\title{
RINGS WITH MANY IDEMPOTENTS
}

\author{
HUANYIN CHEN
}

(Received 31 July 1998)

\begin{abstract}
We introduce a new stable range condition and investigate the structures of rings with many idempotents. These are also generalizations of corresponding results of J. Stock and H. P. Yu.
\end{abstract}

Keywords and phrases. Idempotent 1-stable range, exchange ring, Whitehead group.

1991 Mathematics Subject Classification. Primary 16A30, 16D70.

In this paper, we examine the properties of rings satisfying idempotent 1-stable range and give one large class of such rings. We show that many useful exchange rings belong to the new class of rings. As an application, we also give a new elementwise characterization of strongly $\pi$-regular rings. These are generalizations of many known results.

Throughout, $R$ is an associative ring with identity. $M_{n}(R)$ denotes the ring of $n \times n$ matrices over $R$. Let $M_{n}(R)$ has an identity $I_{n}$, and let its group of units be the general linear group $\mathrm{GL}_{n}(R)$. Set

$$
\begin{gathered}
B_{i j}(x)=I_{2}+x e_{i j} \quad(i \neq j, 1 \leq i, j \leq 2), \\
{[\alpha, \beta]=\alpha e_{11}+\beta e_{22},}
\end{gathered}
$$

where $e_{11}, e_{22}$ and $e_{i j}(i \neq j, 1 \leq i, j \leq 2)$ are all matrix units.

DEFINITION 1. A ring $R$ is said to satisfy idempotent 1-stable range provided that for any $a, b \in R, a R+b R=R$ implies there exists an idempotent $e \in R$ such that $a+b e$ is left invertible in $R$.

Proposition 2. The following are equivalent:

(1) $R$ satisfies idempotent 1-stable range.

(2) For any $a, b \in R, a R+b R=R$ implies there exists an idempotent $e \in R$ such that $a+b e \in U(R)$.

Proof. (2) $\Rightarrow(1)$ is trivial.

$(1) \Rightarrow(2)$ Given $a R+b R=R$. Then there exists an idempotent $e \in R$ such that $a+b e=$ $u$ is left invertible in $R$. Assume that $v u=1$ for some $v \in R$. Then $v R+0 R=R$. Thus, we can find an idempotent $f \in R$ such that $v+0 \cdot f=v$ is left invertible in $R$. So $v$ is a unit, and then $a+b e$ is a unit.

COROLLARY 3. The following are equivalent:

(1) $R$ satisfies idempotent 1-stable range. 
(2) For any $a, b \in R, a R+b R=R$ implies there exists an idempotent $e \in R$ such that $a+b e$ is right invertible in $R$.

Proof. $(1) \Rightarrow(2)$ is clear from Proposition 2.

(2) $\Rightarrow$ (1) Given $a R+b R=R$, then there exists an idempotent $e \in R$ such that $a+b e=$ $u$ is right invertible. Assume that $u v=1$ for some $v \in R$. Since

$$
v R+(1-v u) R=R,
$$

we can find an idempotent $f \in R$ such that

$$
v+(1-v u) f=w
$$

is right invertible in $R$. Obviously,

$$
u w=u(v+(1-v u) f)=1 .
$$

This implies that $w$ is a unit. So $a+b e$ is a unit, as required.

Now we investigate elements in 2-dimensional general linear groups over rings satisfying idempotent 1-stable range. As an application, we shall give an element-wise characterization of such rings.

THEOREM 4. The following are equivalent:

(1) $R$ satisfies idempotent 1-stable range.

(2) For any $A \in \mathrm{GL}_{2}(R)$, there exists an idempotent $e \in R$ such that

$$
A=[*, *] B_{21}(*) B_{12}(*) B_{21}(-e) .
$$

Proof. (1) $\Rightarrow(2)$ Given any $A=\left(a_{i j}\right) \in \mathrm{GL}_{2}(R)$. Then we have

$$
a_{11} R+a_{12} R=R
$$

So we can find an idempotent $f \in R$ such that

$$
a_{11}+a_{12} f=u \in U(R) .
$$

It is easy to verify that

$$
B_{21}\left(-\left(a_{21}+a_{22} f\right) u^{-1}\right) A B_{21}(f) B_{12}\left(-u^{-1} a_{12}\right)=\left[u, a_{22}-\left(a_{21}+a_{22} f\right) u^{-1} a_{12}\right] .
$$

So

$$
A=[*, *] B_{21}(*) B_{12}(*) B_{21}(-f) .
$$

Let $e=-f$. Thus the result follows.

(2) $\Rightarrow(1)$ Given $a R+b R=R$. Then $a x+b y=1$ for some $x, y \in R$. It is easy to verify that

$$
\left(\begin{array}{cc}
a & b y \\
1 & -x
\end{array}\right)=B_{12}(a)\left(\begin{array}{ll}
0 & 1 \\
1 & 0
\end{array}\right) B_{12}(-x) \in \mathrm{GL}_{2}(R) .
$$

So we can find an idempotent $e \in R$ such that 


$$
\left(\begin{array}{cc}
a & b y \\
1 & -x
\end{array}\right)=[*, *] B_{21}(*) B_{12}(*) B_{21}(-e) .
$$

Thus, $a+$ bye $=u \in U(R)$. So we can verify the following.

$$
\left(\begin{array}{cc}
a & b \\
y e & 1
\end{array}\right)=B_{12}(b)\left(\begin{array}{cc}
u & 0 \\
0 & 1
\end{array}\right) B_{21}(y e) \in \mathrm{GL}_{2}(R) .
$$

Consequently, there is an idempotent $f \in R$ such that

$$
\left(\begin{array}{cc}
a & b \\
y e & 1
\end{array}\right)=[*, *] B_{21}(*) B_{12}(*) B_{21}(-f)
$$

and then

$$
\left(\begin{array}{cc}
a & b \\
y e & 1
\end{array}\right) B_{21}(f)=[*, *] B_{21}(*) B_{12}(*) .
$$

Therefore $a+b f \in U(R)$, as desired.

THEOREM 5. The following are equivalent:

(1) $R$ satisfies idempotent 1-stable range.

(2) For any $x, y \in R$, there exists an idempotent $e \in R$ such that $x y-x e+1 \in U(R)$.

Proof. (1) $\Rightarrow(2)$ For any $x, y \in R$,

$$
(1+x y) R+(-x) R=R .
$$

So we can find an idempotent $e \in R$ such that

$$
x y-x e+1=(1+x y)+(-x) e \in U(R) .
$$

(2) $\Rightarrow(1)$ Given $x y+b=1$, there exists an idempotent $e \in R$ such that

$$
(-y) x-(-y) e+1 \in U(R) \text {. }
$$

Let $x-e=a$. Then

$$
1-y a=u \in U(R)
$$

Clearly, we have

$$
x(1-y a)-b a=x-(x y+b) a=x-a=e .
$$

So

$$
x-b a(1-y a)^{-1}=e(1-y a)^{-1} .
$$

From $x y+b=1$, we have

$$
\left(x-b a(1-y a)^{-1}\right) y+b\left(1+a(1-y a)^{-1} y\right)=1 .
$$

Hence,

$$
e(1-y a)^{-1} y+b\left(1+a(1-y a)^{-1} y\right)=1 .
$$

So

$$
e(1-y a)^{-1} y(1-e)+b\left(1+a(1-y a)^{-1} y\right)(1-e)=1-e
$$

and then

$$
e+b\left(1+a(1-y a)^{-1} y\right)(1-e)=1-e(1-y a)^{-1} y(1-e)
$$


Clearly,

$$
1-e(1-y a)^{-1} y(1-e)=\left(1+e(1-y a)^{-1} y(1-e)\right)^{-1} \in U(R) .
$$

So

$$
\begin{aligned}
x+b(-a & \left.(1-y a)^{-1}+\left(1+a(1-y a)^{-1} y\right)(1-e)(1-y a)^{-1}\right) \\
= & x-b a(1-y a)^{-1}+b\left(1+a(1-y a)^{-1} y\right)(1-e)(1-y a)^{-1} \\
& =e(1-y a)^{-1}+b\left(1+a(1-y a)^{-1} y\right)(1-e)(1-y a)^{-1} \\
& =\left(1-e(1-y a)^{-1} y(1-e)\right)(1-y a)^{-1} \in U(R) .
\end{aligned}
$$

Therefore $R$ has stable range one.

Given any $A=\left(a_{i j}\right) \in \mathrm{GL}_{2}(R)$, there are $h, k \in R$ such that

$$
a_{11} h+a_{12} k=1 \text {. }
$$

Since $R$ has stable range one, there exists a $z \in R$ such that

$$
a_{11}+a_{12} z=q \in U(R) .
$$

It is easy to verify that

$$
B_{21}\left(-\left(a_{21}+a_{22} z\right) q^{-1}\right) A B_{21}(z) B_{12}\left(-q^{-1} a_{12}\right)=\left[q, a_{22}-\left(a_{21}+a_{22} z\right) q^{-1} a_{12}\right] .
$$

Obviously,

$$
a_{22}-\left(a_{21}+a_{22} z\right) q^{-1} a_{12} \in U(R),
$$

and then we have $m, n \in R$ such that

$$
A=[*, *] B_{21}(*) B_{12}(m) B_{21}(n) .
$$

So there is an idempotent $f \in R$ such that

$$
1+m(n+1-f)=v \in U(R) .
$$

Let $e=1-f$ and $n=e+s$, then $n=-e$. Consequently, we see that

$$
A=[*, *] B_{21}(*) B_{12}(m) B_{21}(s) B_{21}(-e) .
$$

Since $1+m s \in U(R)$, one can verify

$$
B_{12}(m) B_{21}(s)=[1+m s, 1] B_{21}(s) B_{12}(m)\left[1,(1+s m)^{-1}\right],
$$

whence

$$
A=[*, *] B_{21}(*) B_{12}(*) B_{21}(-e) .
$$

According to Theorem 4, we complete the proof.

As an immediately consequence, we now derive the following result which shows that idempotent 1-stable range property is left-right symmetric. 
COROLLARY 6. The following are equivalent:

(1) $R$ satisfies idempotent 1 -stable range.

(2) For any $a, b \in R, R a+R b=R$ implies there exists an idempotent $e \in R$ such that $a+e b \in U(R)$.

Proof. $R$ satisfies idempotent 1-stable range if and only if for any $x, y \in R$, there exists an idempotent $e \in R$ such that

$$
x y-x e+1=1+x(y-e)=u \in U(R) .
$$

Then a direct computation gives

$$
\begin{aligned}
& (1+(y-e) x)\left(1-(y-e)\left(x+\left(u^{-1}-1\right) x\right)\right) \\
& \quad=\left(1-(y-e)\left(x+\left(u^{-1}-1\right) x\right)\right)(1+(y-e) x)=1,
\end{aligned}
$$

whence we can verify that

$$
x y-x e+1=1+x(y-e) \in U(R)
$$

if and only if

$$
1+(y-e) x \in U(R)
$$

if and only if

$$
x^{0} y^{0}-x^{0} e^{0}+1^{0}=1^{0}+x^{0}\left(y^{0}-e^{0}\right) \in U\left(R^{0}\right) .
$$

Consequently, from Theorem 5 , we see that $R$ satisfies idempotent 1-stable range if and only if so does the opposite ring $R^{0}$. Hence the result follows.

COROLLARY 7. The following are equivalent:

(1) $R$ satisfies idempotent 1-stable range.

(2) For any $A \in \mathrm{GL}_{2}(R)$, there exists an idempotent $e \in R$ such that

$$
A=[*, *] B_{12}(*) B_{21}(*) B_{12}(e) .
$$

Proof. Replacing $A$ by its inverse $A^{-1}$, we know that condition (2) can be seen to be equivalent to the following condition: for any $A \in \mathrm{GL}_{2}(R)$, there exists an idempotent $e \in R$ such that the transpose $A^{t}=B_{12}(-e) B_{21}(*) B_{12}(*)[*, *]$. In view of Theorem 4 , we show that condition (2) is equivalent to the opposite ring $R^{0}$ satisfies idempotent 1-stable range. Using Corollary 6, we obtain the result.

COROLLARY 8. The following are equivalent:

(1) $R$ satisfies idempotent 1-stable range.

(2) Given $a x+b=1$ in $R$. Then there exists an idempotent $e \in R$ such that $a e+b \in$ $U(R)$.

(3) Given $a x+b=1$ in $R$. Then there exists an idempotent $e \in R$ such that ex $+b \in$ $U(R)$.

Proof. (1) $\Rightarrow(2)$ Given $a x+b=1$ in $R$. Then $b R+a R=R$. So there exists an idempotent $e \in R$ such that $a e+b \in U(R)$, as asserted. 
(2) $\Rightarrow(1)$ For any $x, y \in R$, we have

$$
(-x) y+(1+x y)=1
$$

So we can find an idempotent $e \in R$ such that

$$
(-x) e+(1+x y) \in U(R) .
$$

That is,

$$
x y-x e+1 \in U(R) .
$$

Therefore the result follows from Theorem 5 .

$(1) \Leftrightarrow(3)$ is obvious by the left-right symmetry of idempotent 1 -stable range condition.

THEOREM 9. The following are equivalent:

(1) $R$ satisfies idempotent 1-stable range.

(2) $R / J(R)$ satisfies idempotent 1-stable range and idempotents can be lifted modulo $J(R)$.

Proof. $\quad(1) \Rightarrow(2)$ Given any $x+J(R), y+J(R) \in R / J(R)$. Since $R$ satisfies idempotent 1 -stable range, by virtue of Theorem 5 , there is an idempotent $e \in R$ such that $x y-$ $x e+1 \in U(R)$. Thus we have

$$
(x+J(R))(y+J(R))-(x+J(R))(e+J(R))+(1+J(R)) \in U\left(\frac{R}{J(R)}\right)
$$

with

$$
e+J(R)=(e+J(R))^{2} \in \frac{R}{J(R)} .
$$

Using Theorem 5, we show that $R / J(R)$ satisfies idempotent 1-stable range.

Given any $a \in R$. We have $a R+(-1) R=R$. So there exists an idempotent $e \in R$ such that $a-e=u$, and then $a=e+u$. Thus $R$ is a clean ring. By [11, Prop. 1.8, Thm. 1.1], $R$ is exchange. Using [11, Cor. 1.3], we see that idempotents can be lifted modulo $J(R)$.

(2) $\Rightarrow$ (1) Given $a R+b R=R$. Then we have

$$
(a+J(R))\left(\frac{R}{J(R)}\right)+(b+J(R))\left(\frac{R}{J(R)}\right)=\frac{R}{J(R)} .
$$

Since $R / J(R)$ satisfies idempotent 1-stable range, there is an idempotent

$$
e+J(R) \in \frac{R}{J(R)}
$$

such that

$$
(a+J(R))+(b+J(R))(e+J(R)) \in U\left(\frac{R}{J(R)}\right) .
$$

As idempotents can be lifted modulo $J(R)$, we may assume $e=e^{2} \in R$. On the other hand, there is some $v \in R$ such that

$$
v(a+b e)-1 \in J(R) .
$$

Hence $a+b e$ is left invertible, as desired.

EXAMPLE 10. Every local ring satisfies idempotent 1-stable range. 
Proof. Since $R$ is local, $R / J(R)$ is a division ring. Let

$$
S=\frac{R}{J(R)} .
$$

Given $a S+b S=S$ with $a, b \in S$. If $a=0$, then $b S=S$. So $a+b \cdot 1=b$ is right invertible in $S$. If $a \neq 0$, then $a+b \cdot 0=a$ is a unit in $S$. By virtue of Corollary 3, we show that $S=R / J(R)$ satisfies idempotent 1-stable range. Since $R$ is a local ring, idempotents can be lifted modulo $J(R)$. From Theorem 9, the result follows.

In general, every ring satisfying idempotent 1-stable range has stable range one, but the converse is not true as the following shows.

EXAMPLE 11. Let $R=\{m / n \in \mathbb{Q} \mid 2 \nmid n$ and $3 \nmid m(m / n$ in lowest terms $)\}$. Then $R$ is a semilocal ring, while idempotents do not lift modulo $J(R)$. So $R$ has stable range one, but $R$ does not satisfy idempotent 1-stable range from Theorem 9.

Let $R$ be an associative ring with identity 1 . Right $R$-module $A$ is said to have finite exchange property if for every right $R$-module $K$ and any two decompositions,

$$
K=M \oplus N=\bigoplus_{i \in I} A_{i},
$$

where $M_{R} \cong A$ and the index set $I$ is finite, there exist submodules $A_{i}^{\prime} \subseteq A_{i}$ such that

$$
K=M \oplus\left(\bigoplus_{i \in I} A_{i}^{\prime}\right) .
$$

We call a ring $R$ is a (right) weakly $P$-exchange ring if every right $R$-module has finite exchange property (cf. [12]). It is well known that regular rings, right perfect rings and weakly right perfect rings are all weakly $P$-exchange, while there still exist weakly $P$ exchange rings which belong to none of the above classes ([12, Ex. 4.6]). $R$ is called to be exchange if right $R$-module $R$ has finite exchange property. We know that regular rings, $\pi$-regular rings, unital $C^{*}$-algebras of real rank zero, semiperfect rings, left or right continuous rings and clean rings are all exchange. In [16], H. P. Yu proved that every exchange ring with all idempotents central has stable range one. Now we generalize this result as follows.

THEOREM 12. Let $R$ be a ring with all idempotents central. Then the following are equivalent:

(1) $R$ satisfies idempotent 1-stable range.

(2) $R$ is a clean ring.

(3) $R$ is an exchange ring.

Proof. (1) $\Rightarrow(2)$ Given any $a \in R$. From $a R+(-1) R=R$, we have an idempotent $e \in R$ such that $a+(-1) e=u$, and then $a=e+u$. So $R$ is clean.

$(2) \Rightarrow(3)$ is clear from [11, Prop. 1.8, Thm. 2.1].

(3) $\Rightarrow(1)$ Assume that $R$ does not satisfies idempotent 1-stable range. By Proposition 2, there exist $a, b \in R$ with $a R+b R=R$, while $a+b p \notin U(R)$ for any $p=p^{2} \in R$.

Let $\Omega=\{A \mid A$ is a two-sided ideal of $R$ such that $a+b q$ is not a unit modulo $A$ for any $\left.q=q^{2} \in R\right\}$. It is easy to check that $\Omega$ is a nonempty inductive set. By using Zorn's 
lemma, we have a two-sided ideal $Q$ of $R$ such that it is maximal in $\Omega$.

By the maximality of $Q$, we show that $R / Q$ is indecomposable as a ring. Given any $x \in R / Q$. Since $R$ is exchange, so is $R / Q$. By [11, Thm. 1.1], there an idempotent $e \in R / Q$ such that

$$
e \in x\left(\frac{R}{Q}\right), \quad 1-e \in(1-x)\left(\frac{R}{Q}\right),
$$

and an idempotent $f \in R / Q$ such that

$$
f \in\left(\frac{R}{Q}\right) x, \quad 1-f \in\left(\frac{R}{Q}\right)(1-x) .
$$

Since idempotents in $R / Q$ can be lifted modulo $Q$, we may assume that $e$ and $f$ are both central idempotents in $R / Q$. So $e=0$ or $e=1$ and $f=0$ or $f=1$. Thus we see that $x$ or $1-x$ is right invertible in $R / Q$. Similarly, $x$ or $1-x$ is left invertible.

Assume that $x \in R / Q$ is not invertible. If $x$ is not left invertible in $R / Q$, then $r x$ is not left invertible for any $r \in R / Q$. Thus $1-r x$ is left invertible, whence $r x$ is left quasi-regular. This shows that $x \in J(R / Q)$. If $x$ is not right invertible in $R / Q$, similarly to the discussion above, we have $x \in J(R / Q)$. So $J(R / Q)=\{x \in R / Q \mid x$ is not invertible in $R / Q\}$. This implies that $R / Q$ is local. By virtue of Example 10, we claim that $R / Q$ satisfies idempotent 1-stable range, a contradiction. Hence the result follows.

Theorem 12 shows that exchange rings with all idempotents central satisfy idempotent 1-stable range. Now we give an exchange $\operatorname{ring} R$ with noncentral idempotents, while it indeed satisfy idempotent 1-stable range.

EXAMPLE 13. Let

$$
R=\left(\begin{array}{cc}
\mathbb{Z} / 2 \mathbb{Z} & \mathbb{Z} / 2 \mathbb{Z} \\
0 & \mathbb{Z} / 2 \mathbb{Z}
\end{array}\right) .
$$

By [17, Ex. 3.10], $R$ is an exchange ring with noncentral idempotents. According to Theorem 5 , we directly verify that $R$ satisfies idempotent 1 -stable range.

Recall that a ring $R$ is said to be strongly $\pi$-regular if every descending chain of right ideals of the form

$$
a R \supseteq a^{2} R \supseteq a^{3} R \supseteq \cdots, \quad a \in R
$$

becomes stationary. It is well known that every strongly $\pi$-regular ring is clean. Now we generalize this fact as follows.

COROLLARY 14. Let $R$ be a strongly $\pi$-regular ring. If $x, y \in R$ with $x y=y x$, then there exists an idempotent $e \in R$ such that

$$
x y+x e+1 \in U(R) .
$$

Proof. Given any $x, y \in R$ with $x y=y x$. Let $S$ be an additive subgroup generated by the set

$$
\left\{x^{m} y^{n} \mid m, n \geq 0\right\} \text {. }
$$


Then $S$ is a commutative subring of $R$. By virtue of [3, Cor. 1.10], we can find a commutative strongly $\pi$-regular subring $T$ of $R$ which contains $S$.

By Theorem 12, $T$ satisfies idempotent 1-stable range with $x, y \in T$. Thus we can find

$$
f=f^{2} \in T \subseteq R
$$

such that

$$
x(y+1)-x f+1 \in U(T) \subseteq U(R) .
$$

Let $e=1-f$. Then we have idempotent $e=e^{2} \in R$ such that $x y+x e+1 \in U(R)$, as desired.

A ring $R$ is said to be right (left) quasi-duo if every maximal right (left) ideal is twosided. By an argument of $\mathrm{H}$. P. Yu, every weakly $P$-exchange ring with all idempotents central is right (left) quasi-duo. In general, the converse is not true such as

$$
R=\left(\begin{array}{ll}
F & F \\
0 & F
\end{array}\right), \quad \text { where } F \text { is a field. }
$$

Now we give a theorem which guarantees the existence of one large class of rings satisfying idempotent 1-stable range.

THEOREM 15. Let $R$ be a right or left quasi-duo weakly $P$-exchange ring. Then $R$ satisfies idempotent 1-stable range.

Proof. By [15, Prop. 2.1(1)], right primitive right quasi-duo rings are division. So every right or left quasi-duo ring has primitive factors artinian. Let $Q$ be a prime ideal of $R$. Since $R$ is a weakly $P$-exchange ring, so is $R / Q$. Similarly to [12, Prop. 4.1(2)], the finite exchange property of $R^{(\mathbb{N})}$ forces $J(R / Q)$ to be $T$-nilpotent. Assume that

$$
0 \neq a \in J\left(\frac{R}{Q}\right) .
$$

Then there exist $x_{1}, x_{2}, \ldots, x_{n}, \ldots \in R / Q$ such that

$$
a x_{1} a \neq 0, \quad a x_{2} a x_{1} a \neq 0, \ldots, \quad a x_{n} \cdots a x_{1} a \neq 0, \ldots,
$$

a contradiction. Thus $J(R / Q)=0$. So $R / Q$ is an indecomposable exchange ring with primitive factors artinian and $J(R / Q)=0$. Using [17, Lem. 3.7], we claim that $R / Q$ is simple artinian. Thus $R$ is an exchange ring with prime factors artinian, so it is strongly $\pi$-regular. Using [17, Thm. 3.8], we see that $R / J(R)$ is a regular ring with all idempotents central. From Theorem $12, R / J(R)$ satisfies idempotent 1-stable range. As idempotents can be lifted modulo $J(R)$, we obtain the result from Theorem 9 .

Recall that $p(a)=a, p(a, b)=1+a b$ and $p(a, b, c)=a+c+a b c$ for any $a, b, c \in R$. $W(R)$ denotes the subgroup of $U(R)$ generated by

$$
\left\{p(a, b, c) p(c, b, a)^{-1} \mid p(a, b, c) \in U(R), a, b, c \in R\right\},
$$


and $V(R)$ denotes the subgroup of $U(R)$ generated by

$$
\left\{p(a, b) p(b, a)^{-1} \mid p(a, b) \in U(R), a, b \in R\right\} .
$$

It is easy to verify that

$$
p(a, b, c)=p(a, b) c+p(a), p(a, b, c) p(b, a)=p(a, b) p(c, b, a)
$$

and

$$
\left(\begin{array}{cc}
* & * \\
p(a, b, c) & *
\end{array}\right)=B_{21}(a) B_{12}(b) B_{21}(c) .
$$

We end this note by investigating Whitehead groups of rings with many idempotents.

TheOREM 16. Let $R$ satisfy idempotent 1-stable range. Then

$$
K_{1}(R) \cong \frac{U(R)}{V(R)} .
$$

Proof. For any $a, b, c \in R$ with $p(a, b, c) \in U(R)$, we see that $p(c, b, a) \in U(R)$. By virtue of Theorem 5 , there exists an idempotent $e \in R$ such that $1+b(c-e) \in U(R)$. Let $c-e=t$. Then $c=t+e$ and $1+b t \in U(R)$. Observing that

$$
\begin{aligned}
\left(\begin{array}{cc}
* & * \\
p(a, b, c) & *
\end{array}\right) & =B_{21}(a) B_{12}(b) B_{21}(c) \\
& =\left(B_{21}(a) B_{12}(b) B_{21}(t)\right) B_{21}(e) \\
& =B_{21}(a)[1+b t, 1] B_{21}(t) B_{12}(b)\left[1,(1+t b)^{-1}\right] B_{21}(e) \\
& =[1+b t, 1] B_{21}(a+t+a b t) B_{12}(b)\left[1,(1+t b)^{-1}\right] B_{21}(e) \\
& =\left[1+b t,(1+t b)^{-1}\right] B_{21}((1+t b)(a+t+a b t)) B_{12}\left(b(1+t b)^{-1}\right) B_{21}(e) \\
& =\left(\begin{array}{cc}
* & * \\
(1+t b)^{-1} p\left((1+t b)(a+t+a b t), b(1+t b)^{-1}, e\right) & *
\end{array}\right) .
\end{aligned}
$$

Thus we have

$$
p(a, b, c)=(1+t b)^{-1} p\left((1+t b)(a+t+a b t), b(1+t b)^{-1}, e\right) .
$$

Analogously to [10, Thm. 1.6], we know that

$$
\begin{aligned}
p(a, b, c) & \equiv(1+t b)^{-1} p\left(e, b(1+t b)^{-1},(1+t b)(a+t+a b t)\right)(\bmod V(R)) \\
& =(1+t b)^{-1}\left(p\left(e, b(1+t b)^{-1}\right)(1+t b)(a+t+a b t)+p(e)\right) \\
& =(1+t b)^{-1}\left(p\left(e, b(1+t b)^{-1}\right) p(t, b) p(a, b, t)+p(e)\right) \\
& =(1+t b)^{-1}\left(p\left(e, b(1+t b)^{-1}\right) p(t, b, a) p(b, t)+p(e)\right) .
\end{aligned}
$$

Similarly, we can verify that

$$
\begin{aligned}
p(c, b, a) & =p\left(e,(1+b t)^{-1} b,(t+a+t b a)(1+b t)\right)(1+b t)^{-1} \\
& =\left(p\left(e,(1+b t)^{-1} b\right)(t+a+t b a)(1+b t)+p(e)\right)(1+b t)^{-1} \\
& =\left(p\left(e,(1+b t)^{-1} b\right) p(t, b, a) p(b, t)+p(e)\right)(1+b t)^{-1} .
\end{aligned}
$$


It is easy to check that

$$
b(1+t b)^{-1}=(1+b t)^{-1} b .
$$

Consequently, we have

$$
(1+t b) p(a, b, c) \equiv p(c, b, a)(1+b t)(\bmod V(R)) .
$$

Thus

$$
p(a, b, c)(p(c, b, a))^{-1} \in V(R) .
$$

Therefore we conclude that

$$
K_{1}(R) \cong \frac{U(R)}{W(R)} \cong \frac{U(R)}{V(R)},
$$

as asserted.

\section{REFERENCES}

[1] P. Ara, Strongly $\pi$-regular rings have stable range one, Proc. Amer. Math. Soc. 124 (1996), no. 11, 3293-3298. MR 97a:16024. Zbl 865.16007.

[2] A. Badawi, On Abelian $\pi$-regular rings, Comm. Algebra 25 (1997), no. 4, 1009-1021. CMP 97 09. Zbl 881.16003.

[3] W. D. Burgess and P. Menal, On strongly $\pi$-regular rings and homomorphisms into them, Comm. Algebra 16 (1988), no. 8, 1701-1725. MR 89f:16015. Zbl 655.16006.

[4] V. P. Camillo and H. P. Yu, Exchange rings, units and idempotents, Comm. Algebra 22 (1994), no. 12, 4737-4749. MR 95d:16013. Zbl 811.16002.

[5] _ Stable range one for rings with many idempotents, Trans. Amer. Math. Soc. 347 (1995), no. 8, 3141-3147. MR 95j:16006. Zbl 848.16008.

[6] R. Camps and P. Menal, Power cancellation for Artinian modules, Comm. Algebra 19 (1991), no. 7, 2081-2095. MR 92m:16006. Zbl 732.16016.

[7] H. Chen, Comparability of modules over regular rings, Commun. Algebra 25 (1997), no. 11, 3531-3543. Zbl 887.16001.

[8] _ Elements in one-sided unit regular rings, Comm. Algebra 25 (1997), no. 8, 25172529. CMP 97 15. Zbl 881.16004.

[9] K. R. Goodearl and P. Menal, Stable range one for rings with many units, J. Pure Appl. Algebra 54 (1988), no. 2-3, 261-287. MR 89h:16011. Zbl 653.16013.

[10] P. Menal and J. Moncasi, $K_{1}$ of von Neumann regular rings, J. Pure Appl. Algebra 33 (1984), no. 3, 295-312. MR 86i:18014. Zbl 541.16021.

[11] W. K. Nicholson, Lifting idempotents and exchange rings, Trans. Amer. Math. Soc. 229 (1977), 269-278. MR 55 12757. Zbl 352.16006.

[12] J. Stock, On rings whose projective modules have the exchange property, J. Algebra 103 (1986), no. 2, 437-453. MR 88e:16038. Zbl 603.16016.

[13] L. N. Vaserstein, Bass's first stable range condition, J. Pure Appl. Algebra 34 (1984), no. 2-3, 319-330, Proceedings of the Luminy conference on algebraic $K$-theory. MR 86c:18009. Zbl 547.16017.

[14] H. You, Stable rings and two-dimensional linear groups over them, Chinese Ann. Math. Ser. A 7 (1986), no. 3, 255-265 (Chinese). MR 87k:16022. Zbl 644.16012.

[15] H. P. Yu, On quasi-duo rings, Glasgow Math. J. 37 (1995), no. 1, 21-31. MR 96a:16001. Zbl 819.16001.

[16] _ Stable range one for exchange rings, J. Pure Appl. Algebra 98 (1995), no. 1, 105109. MR 96g:16006. Zbl 837.16009. 
[17] _ On the structure of exchange rings, Comm. Algebra 25 (1997), no. 2, 661-670. MR 97k:16010. Zbl 873.16007.

Chen: Department of Mathematics, Hunan Normal University, Changsha 410006, CHINA

E-mail address: chyzx1@sparc2. hunnu.edu.cn 


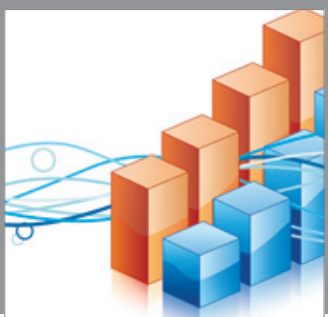

Advances in

Operations Research

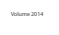

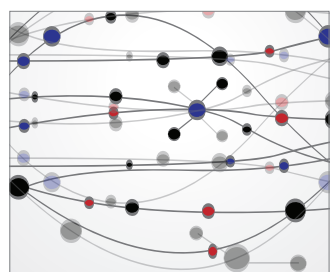

\section{The Scientific} World Journal
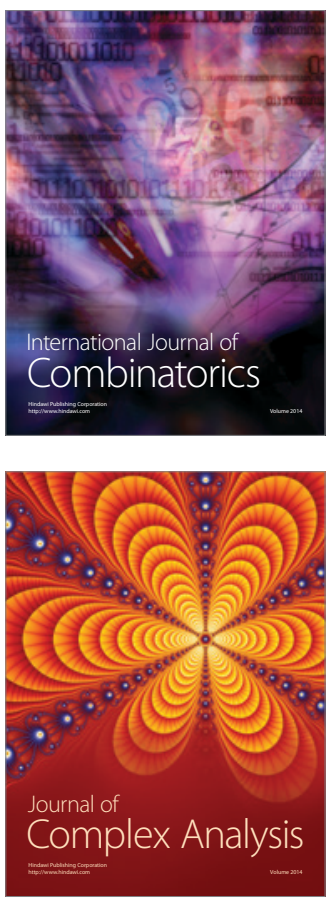

International Journal of

Mathematics and

Mathematical

Sciences
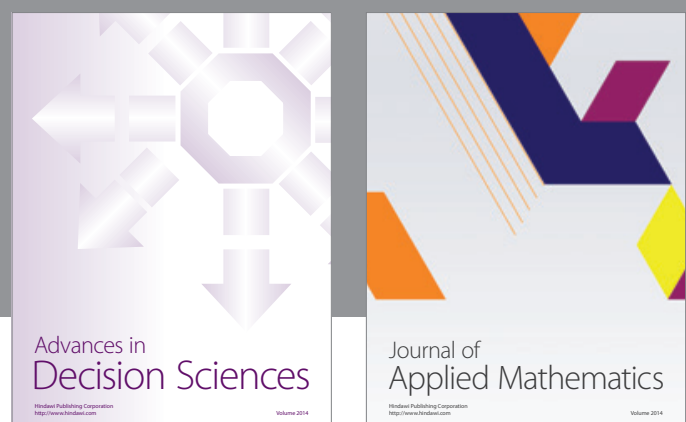

Journal of

Applied Mathematics
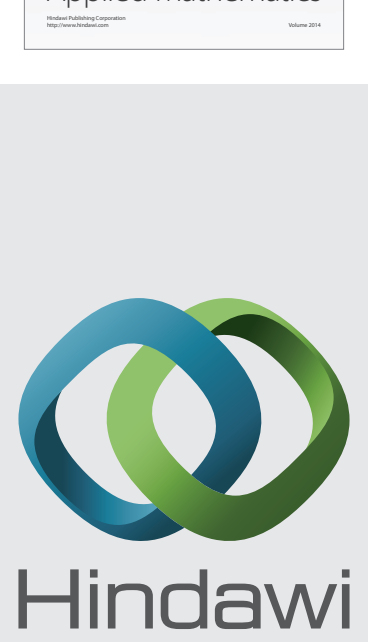

Submit your manuscripts at http://www.hindawi.com
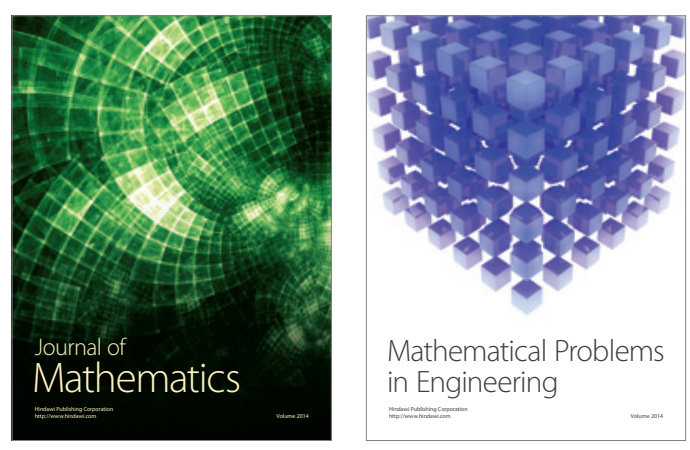

Mathematical Problems in Engineering
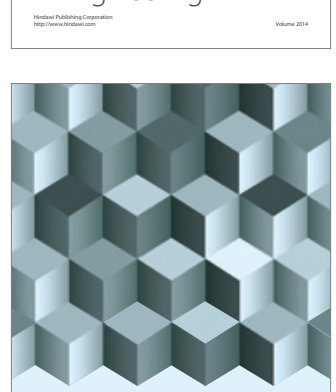

Journal of

Function Spaces
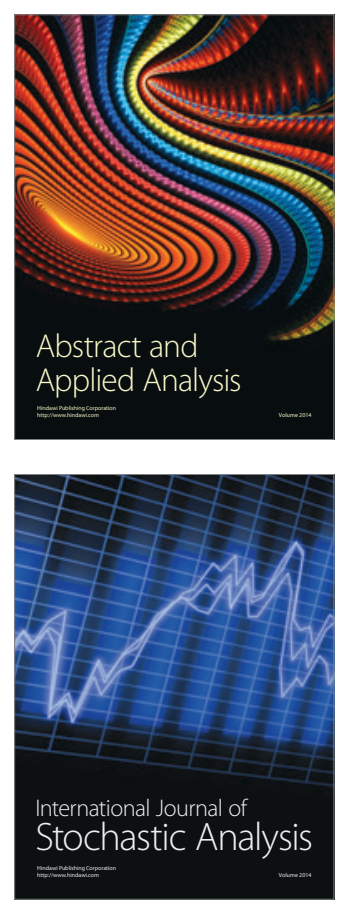

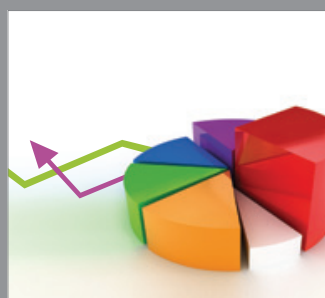

ournal of

Probability and Statistics

Promensencen
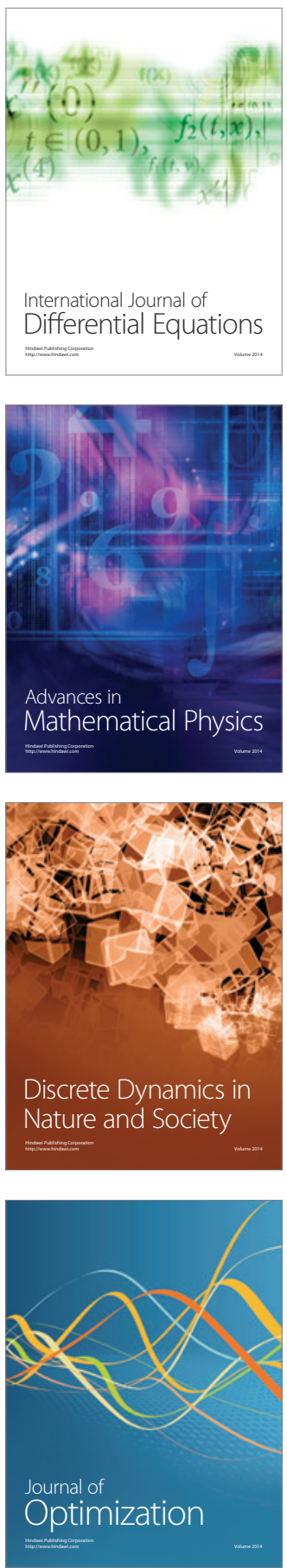